\title{
A Degradation Condition Assessment Technique Based on online ISSE Degradation Feature and Logistic Regression Model
}

\author{
Cancan Wang, Bing Wang, Xiong Hu, Wei Wang and Dejian Sun \\ Logistics Engineering College, Shanghai Maritime University, Shanghai, 201306 China. \\ E-mail: 1002624905@shmtu.edu.cn
}

\begin{abstract}
(Received 1 October 2020; accepted 4 July 2021)
A degradation assessment technique based on an online improved symbol sequence entropy (online ISSE) and a logistic regression model is proposed in this paper. Firstly, the threshold factor is introduced to retain the 'coarse graining' information of direction changing and amplitude information, the 'sensitivity' of improved symbol sequence entropy (SSE) to impact components is reduced and improved symbol sequence entropy (ISSE) is proposed. Then, a sliding window and Weibull distribution theory are used to effectively filter out the influence of fluctuations in the ISSE feature sequence, forming the degradation feature named online_ISSE. Finally, a logistic regression model is trained and constructed, and the health factor $C V$ is calculated online to assess the degradation condition of the unknown signal samples. The lifetime vibration signal of the hoisting gearbox monitored from \#8114 quay crane of the Shanghai Port Container Terminal is introduced for instance analysis. The results show that the proposed $I S S E$ has a better effect in describing the complexity pattern than the $S S E$ algorithm and that the degradation condition can be tracked and assessed accurately based on the technique proposed.
\end{abstract}

\section{INTRODUCTION}

The quay crane, also known as the quayside container crane or bridge crane, is a large harbor hoisting crane used for loading and unloading containers at harbors. Among the main components of the quay crane, the hoisting mechanism is the important power mechanism which is generally composed of a driving motor, a hoisting gearbox, a coiling block and a wire rope. The quay crane usually works under special operational conditions in the harsh environment full of sea breeze and noise. In special periodic hoisting operations, a heavy container with high speed will bring about large impact on the hoisting mechanism in the form of quick start and stop occur, or the container frequently passing by the track hinge point. Performance degradation or even sudden failure will occur under the long-term influence described above, ${ }^{1}$ leading to downtime or even casualties. At present, the hoisting gearbox has become a typical vulnerable part and a health weakness in the quay crane health management. The degradation trend can be tracked accurately on the condition that the vibration signals are monitored online and analyzed effectively, which will lay a foundation for Condition-Based Maintenance (CBM) and improve the safety and reliability of the quay crane. ${ }^{2}$

Feature extraction, which is the foundation of the degradation condition assessment, is the critical step in CBM. The main target of feature extraction is the mining health indicator which is able to reflect a performance degradation trend quantitatively and accurately. At present, linear analysis methods including time domain, frequency domain and time-frequency domain are widely used. ${ }^{3-5}$ Some typical indicators proposed include spectral kurtosis, ${ }^{6,7}$ spectral L2/L1 norm, ${ }^{8}$ kurtosis ${ }^{9}$ etc. Considering the nonlinear and non-stationary character of mechanical vibration signals, a complexity analysis method is used based on information from entropy and fractal dimension and is usually employed in feature analysis of rotating machinery such as bearings and gears in recent years, such as modified multi-scale symbolic dynamic entropy, ${ }^{10}$ LMDsample entropy, ${ }^{11}$ multi scale permutation entropy, ${ }^{12,13}$ morphological fractal dimension ${ }^{14}$ etc.

Some previous studies have shown that vibration signals of the quay crane have a certain degree of complexity character. However, the accurate complexity value reflecting the degradation condition is hard to be extracted because of the random shock components in the signal caused by the environment and operation conditions. As a "coarse grain" signal analysis method, ${ }^{15}$ symbol dynamics analysis is able to process time series and preserve the direction exchanging information with symbolic methods. The SSE has been successfully applied in electroencephalogram (EEG) signal analysis ${ }^{16-18}$ these studies have proven the advantages including simple calculation, fast calculation speed and strong anti-interference ability. At present, the SSE algorithm is rarely used in the fault prognostic field of mechanical equipment. Considering the complexity and large impact character of the quay crane vibration signal, the 'coarse graining' advantage in amplitude processing of SSE will be employed in this paper for the degradation feature extraction.

On the basis of feature extraction, the degradation condition assessment is studied for assessing the performance degradation condition through the degradation feature indicators. Current studies mainly focus on data-based assessment methods in three aspects as follows. The first type is based on probability similarity including the Gaussian Mixture Model (GMM), ${ }^{19}$ and the Hidden Markov model (HMM) ${ }^{20}$ The second type is based a on reconstruction model such as the logistic regression model. ${ }^{21}$ The third method is mainly based on the boundary model including Markov distance, ${ }^{22}$ fuzzy clustering ${ }^{23}$ etc. Among these methods above, the logistic regression model is able to reconstruct the model with training samples. The model 
offset of the samples will be calculated to assess the degradation condition, the advantages are reflected in simple principle and fast calculation speed. This model is suitable for the dichotomy of health and failure condition, and additionally, the model offset can characterize degradation degree quantitatively. The logistic regression model is employed in the degradation condition assessment considering the advantages above.

In summary, an online degradation condition assessment technique is proposed based on the online improved symbol sequence entropy (online_ISSE) and logistic regression model. The ISSE method is proposed by introducing the threshold factor based on SSE. The degradation feature named online_ISSE is featured with a sliding window and the Weibull distribution theory. The online degradation condition can be evaluated combined with the logistic regression model. A lifetime vibration signal of a hoisting gearbox is introduced for instance analysis.

The paper is organized as follows: Section 2 introduces basic theory including the symbol sequence entropy, the Weibull distribution, and the logistic regression model. Section 3 proposes improvement on SSE and expounds ISSE. The procedure of the degradation condition assessment technique is expounded in Section 4. In Section 5, the technique is verified, and the results are discussed. Finally, the conclusion of this paper is given in Section 6.

\section{BASIC THEORY}

\subsection{Symbolic Sequence Entropy}

The $S S E$ is proposed and mainly applied in EEG signal analysis. The basic principle is as follows. ${ }^{24}$

Assuming that $R$ is a non-stationary random EEG signal of length $N$. Firstly, the signal is symbolized as Eq. (1) and the changing direction information of the EEG signal is retained by the three symbols including 0,1 , and 2 .

$$
x(i)= \begin{cases}0, & R(i+1)<R(i) \\ 1, & R(i+1)=R(i) . \\ 2, & R(i+1)>R(i)\end{cases}
$$

Secondly, a vector $X(i)$ of length $m$ is constructed with the sliding window method in Eq. (2). The possible symbol patterns in $X(i)$ will reach a total of $M=3^{m}$ because of the three symbols defined above.

$$
\begin{aligned}
X(i)=[x(i), x(i+1), \ldots, x(i+(m-1))], & \\
& i=1,2 \ldots, N-m .
\end{aligned}
$$

Thirdly, the probability of each symbol pattern in $X(i)$ is calculated as Eq. (3) where $N_{j}$ represents the number of each symbol pattern.

$$
p_{j}=\frac{N_{j}}{N-m}, \quad j=1,2, \ldots, M .
$$

In the end, the normalized $S S E$ is calculated according to Eq. (4) which should satisfy $N>3^{m}$ generally.

$$
\operatorname{SSE}(m)=\frac{-\sum_{j=1}^{M} p_{j} \lg p_{j}}{\lg M} .
$$

\subsection{Weibull Distribution}

The Weibull distribution is recognized as the most suitable distribution for reliability analysis in engineering. ${ }^{25,26}$ This distribution has been widely used in failure analysis on machinery, electronics, chemicals and materials. The commonly used methods include the single parameter, the two-parameter and the three-parameter model. The two-parameter model is employed in this paper for degradation feature analysis.

The cumulative density function (degree of failure) of the two-parameter Weibull distribution is as follows ${ }^{27}$ :

$$
F(t)=1-\exp \left\{-\left[\frac{t}{\eta}\right]^{\beta}\right\}(t \geq 0)
$$

where $\nu$ means the scale parameter and $\beta$ means the shape parameter. The reliability is defined as follows:

$$
R(t)=1-F(t)=\exp \left\{-\left[\frac{t}{\eta}\right]^{\beta}\right\}(t \geq 0) .
$$

\subsection{Logistic Regression Model}

The logistic regression theory is a multi-parameter statistical method in probability statistics. This theory is able to describe the optimal mapping relationship between a set of multivariate and a set of dichotomous variables. ${ }^{28}$ For any independent variable, the probability $P$ of the response variable will increase according to the S-curve with the independent variable.

The mathematical expression of logistic regression is as follows: 29

$$
P_{i}\left(y_{i}=1 \mid x_{i}\right)=\frac{e^{\alpha+\sum \beta_{i} x_{i}}}{1+e^{\alpha+\sum \beta_{i} x_{i}}}
$$

where $x_{i}$ is a $k$-dimensional independent variable, $y_{i}$ is the observed response variable. The equation of $y_{i}=1$ means the event (device performance degradation) occurs while $y_{i}=0$ means the event does not occur. $P_{i}$ represents the occurring probability of the $i^{\text {th }}$ event, $\alpha$ is the regression intercept and $\beta$ is the regression coefficient.

\section{IMPROVED SYMBOLIC SEQUENCE ENTROPY}

As introduced above, the symbol's type is determined by the magnitude difference of adjacent points in SSE which reflects the changing direction of the signal with symbols 1, 2, 3 sensitively. However, there are many noise and random shock components in the online vibration signal of the quay crane. The components will affect the $S S E$ value sensitively leading to an inaccurate result of complexity pattern in the signal. Therefore, it is necessary to improve the symbol pattern for reducing the sensitivity to direction changes.

In response to this issue, threshold factor $a$ is introduced to keep the changing direction information as well as the amplitude changing information coarsely. The improved symbolic pattern is as follows where five symbols are introduced, and the division pattern is improved. 


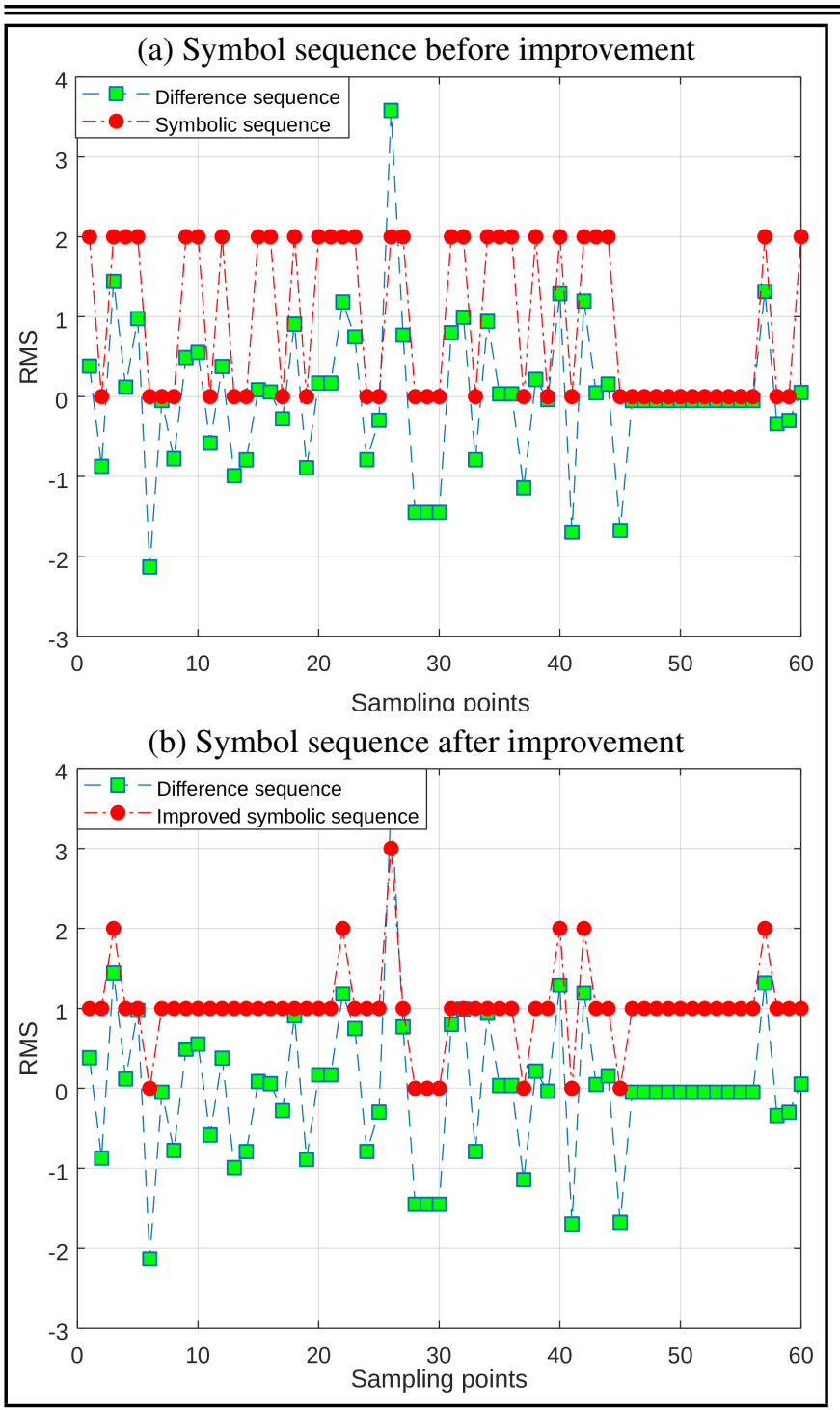

Figure 1. Comparison of different symbol effect.

$$
x(i)=\left\{\begin{array}{lr}
3, & R(i+1)-R(i) \geq 3 a \\
1, & a \leq R(i+1)-R(i)<3 a \\
2, & -a \leq R(i+1)-R(i)<a \\
0, & -3 a \leq R(i+1)-R(i)<a \\
4, \quad R(i+1)-R(i)<-3 a
\end{array} .\right.
$$

The threshold factor determines the coarse degree of the symbolic pattern division. Considering the pattern stability in the degradation condition signal, the threshold factor is set as twice the standard deviation for the degradation condition signal.

A typical vibration $R M S$ sequence of the hoisting gearbox as an example, assuming that the threshold factor is set as $a=1.0$, the symbol effect comparison is shown in Fig. 1. It is evident that the sampling points in the difference sequence whose amplitude is within $|a|$ are all symbolized as 2 by introducing threshold factor, and the amplitude information is also retained coarsely with five symbols. The sensitivity of the improved symbol sequence is reduced which enables the pattern changing law inside the signal to be described.

After the operation of improved symbol process, constructing vectors and calculating probability of each symbol pattern as Eq. (2) and Eq. (3) were completed. In the end, the improved symbol sequence entropy (ISSE) can be calculated according to Eq. (6).

$$
\operatorname{ISSE}(m)=\frac{-\sum_{j=1}^{M} p_{j} \lg p_{j}}{\lg M}
$$

\section{DEGRADATION CONDITION ASSESSMENT BASED ON ONLINE ISSE AND LR MODEL}

Based on the ISSE algorithm, a degradation condition assessment technique based the online improved symbol sequence entropy (online_ISSE) and the logistic regression (LR) is proposed aiming at the special operation condition for the quay crane. The flow chart is shown in Fig. 2.

The following five key steps are included in the Fig. 2.

(1) Online monitoring of vibration signals. The condition monitoring system of the quay crane is used as a platform to collect vibration signals continuously. Considering the massiveness and low density of the actual monitoring signals, the vibration $R M S$ sequence Data $=x_{i}(i=1,2, \ldots)$ from the hoisting gearbox of the quay crane is adopted in reality.

(2) Feature extraction based on ISSE. The vibration $R M S$ sequence is divided into analysis group $G_{j}(j=1,2, \ldots)$ by setting analysis period $T$. The division pattern is as follows:

$$
\begin{aligned}
& G_{1}=\left[x_{1}, x_{2}, \ldots, x_{T}\right] \\
& G_{2}=\left[x_{T+1}, x_{T+2}, \ldots x_{2 T}\right] \\
& \vdots \\
& G_{j}=\left[x_{(j-1) T+1}, x_{(j-1) T+2}, \ldots, x_{j T}\right] .
\end{aligned}
$$

The calculating threshold factor $a$ and extracting ISSE value of each group, obtaining the $I S S E$ feature sequence $I_{j}$.

(3) Online ISSE feature calculation. The sliding window width $w$ and step size $s$ are set to obtain sliding window $W_{q}$ $(q=1,2, \ldots)$, and each sliding window is an ISSE sequence with the number of $w$. The pattern is as follows:

$$
\begin{aligned}
& W_{1}=\left[I_{1}, I_{2}, \ldots, I_{w}\right] ; \\
& W_{2}=\left[I_{1+s}, I_{2+s}, \ldots, I_{s+w}\right] ; \\
& W_{3}=\left[I_{1+2 s}, I_{2+2 s}, \ldots, I_{2 s+w}\right] ; \\
& \vdots \\
& W_{q}=\left[I_{1+(q-1) s}, I_{2+(q-1) s}, \ldots, I_{(q-1) s+w}\right] .
\end{aligned}
$$

The Weibull distribution fitting is performed on each sliding window ${ }^{18}$ and the scale parameter will be obtained which serves as an online degradation feature online $I S S E_{q}$ of the window. Moving the sliding window with $s$ step and calculating online degradation feature curve online ISSE which is used to track the online degradation character of key components in the quay crane.

(4) Logistic regression model construction. Taking typical health and failure samples as training data, the health indicator $C V$ are defined as follows:

$$
C V=1-P=1-\frac{e^{\alpha+\sum \beta_{i} x_{i}}}{1+e^{\alpha+\sum \beta_{i} x_{i}}}=\frac{1}{1+e^{\alpha+\sum \beta_{i} x_{i}}} .
$$




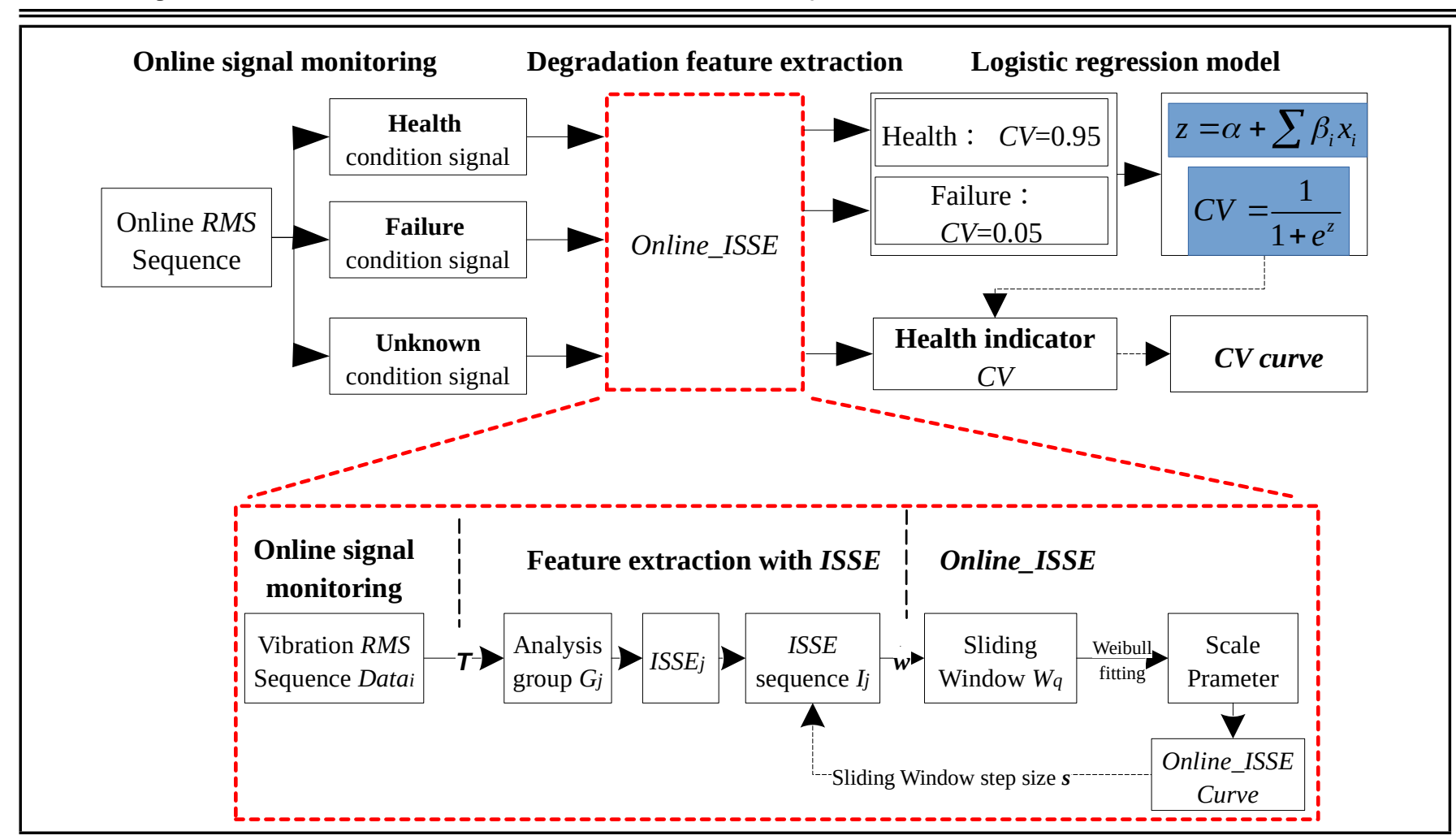

Figure 2. Flow chart of degradation condition assessment.

Table 1. Basic specifications of \#8114 quay crane.

\begin{tabular}{||l|l|l|l||}
\hline Number & Entry & Condition & Specification \\
\hline \multirow{2}{*}{1} & \multirow{2}{*}{ Rated loading } & Under the spreader & $50 \mathrm{t}$ \\
\cline { 3 - 4 } & & Under the hanger & $62.5 \mathrm{t}$ \\
\hline 2 & Gauge & & $16 \mathrm{~m}$ \\
\hline \multirow{2}{*}{3} & \multirow{2}{*}{ Hoisting height } & Rail surface & $10 \mathrm{~m}$ \\
\cline { 3 - 4 } & & Under the rail & $36 \mathrm{~m}$ \\
\hline \multirow{2}{*}{4} & \multirow{2}{*}{ Hoisting speed } & Full loading & $50 \mathrm{~m} / \mathrm{min}$ \\
\cline { 3 - 4 } & & Empty loading & $120 \mathrm{~m} / \mathrm{min}$ \\
\hline
\end{tabular}

In this paper, $C V$ is set as 0.95 for the health samples while $C V$ is set as 0.05 for the samples in the failure condition. The processing model training and calculating the parameters $\alpha$ and $\beta$, establish the $L R$ based degradation assess model.

(5) Degradation condition assessment. As to the unknown condition samples, the vibration $R M S$ sequence as step (2)-(3) and obtaining online_ISSE will be processed. After inputting the degradation feature to the constructed $L R$ model, the value of $C V$ will be obtained to assess the degradation condition.

\section{THE QUAY CRANE HOISTING GEARBOX DEGRADATION CONDITION ASSESSMENT}

\subsection{Lifetime Vibration Dataset of Hoisting Gearbox}

An instance analysis is carried out using the lifetime vibration signal of the hoisting gearbox. The dataset is monitored from the \#8114 quay crane of Shanghai Port Container Terminal. The equipment type is $50 t-22 m$ Shore Rail Container Crane. An actual example is shown in Fig. 3. The basic specifications are shown in Table 1.

We began to monitor this equipment by means of vibration, temperature and stress at more than forty measuring points based on Net-CMAS system since 2007 when the quay crane

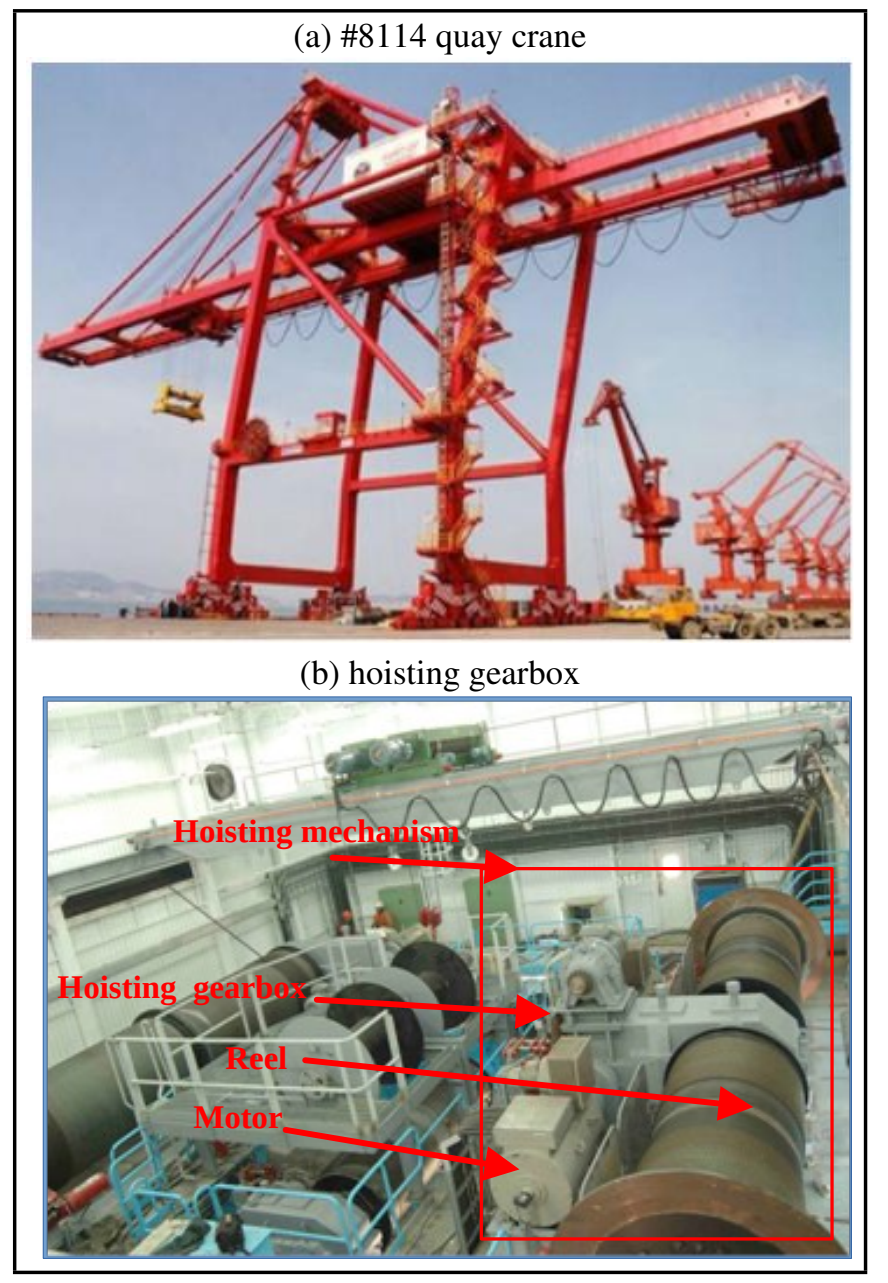

Figure 3. Actual appearance of $\# 8114$ and hoisting gearbox. 


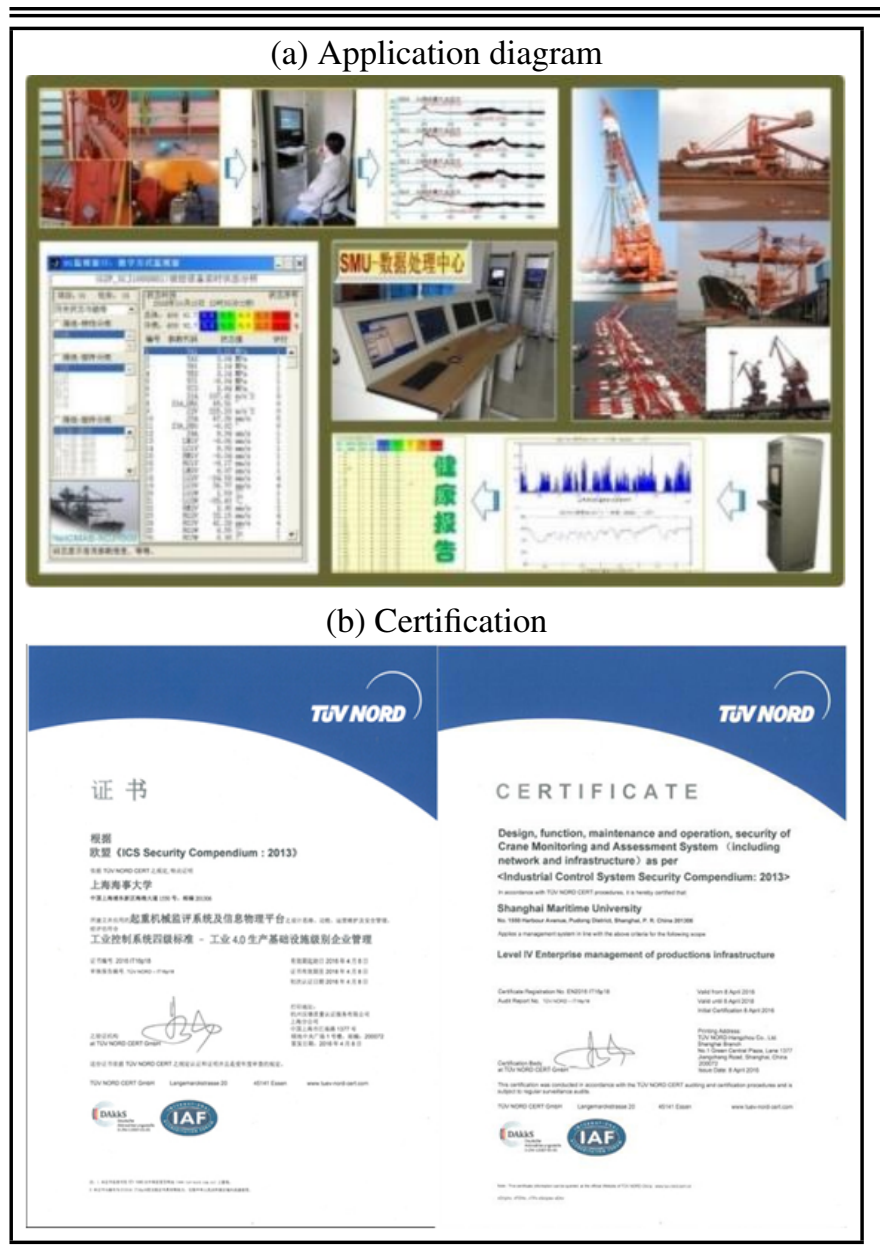

Figure 4. Description of the Net-CMAS system.

was installed. The applied Net-CMAS system was developed by our team which has met EU Industrial Control System Outline Specification in terms of function, performance and safety management. ${ }^{30}$ This system, which is a typical application example of China Manufacturing 2025 Industrial Control System Automation, has reached ICS Compendium Industry 4.0 Level 4 standard. The application and certificate are shown in Fig. 4.

More than forty measuring points of the quay crane are equipped with vibration, temperature sensor and strain gauges. An industrial computer is installed in the control room to accomplish collection and storage of the online signal. The vibration sensor at vertical direction of high-speed output shaft of the hoisting gearbox is shown in Fig. 5.

The sampling frequency is $24 \mathrm{kHz}$ and sampling time is 1 second. The sampling interval is set as 10 seconds. The system calculates and stores effective value $R M S$ of each sampling signal, obtaining vibration $R M S$ sequence online. After 7 years and 8 months, the Net-CMAS system's alarm went off at the high-speed input shaft of the hoisting gearbox. The failure occurred on the input shaft roller bearing and the failure mode is roller abrasion. The worn roller is shown in Fig. 6.

The non-operating data such as a shutdown condition is filtered out automatically, forming a lifetime hoisting gearbox $R M S$ sequence. The time domain waveform is shown in Fig. 7. It is evident that the amplitude is constantly increasing as a whole trend and there are a wide range of impact components. The amplitude drops rapidly after the inspection point. It is apparent that the impact components of the $R M S$ sequence are frequent and large. It is difficult to track the performance

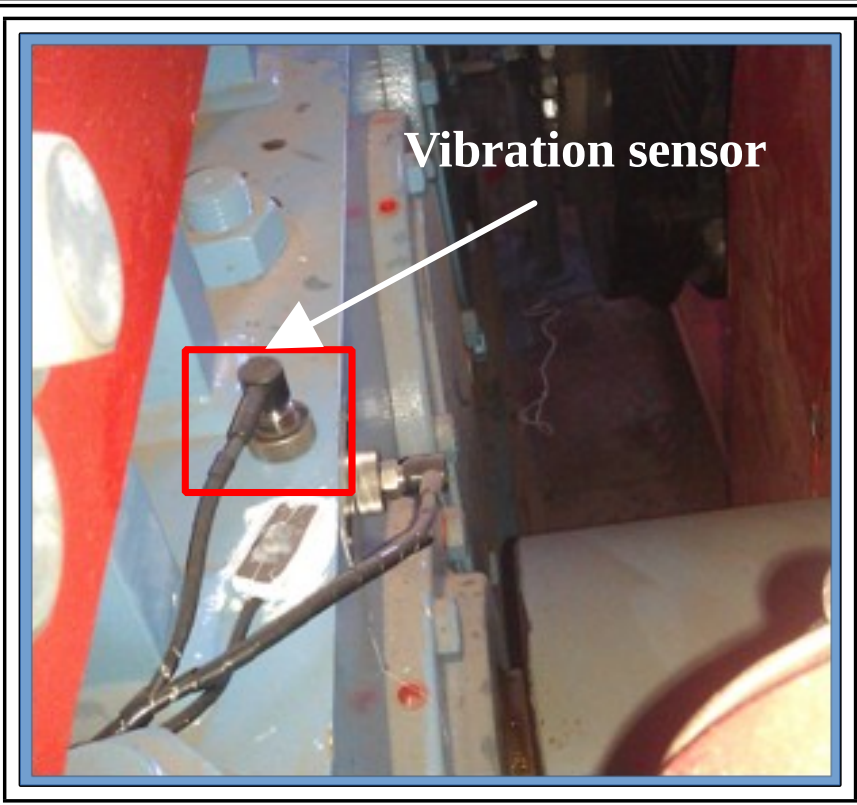

Figure 5. Vibration sensors at hoisting gearbox.

degradation condition accurately and can even cause misjudgment. It is necessary to perform accurate degradation feature analysis.

\subsection{ISSE Analysis}

When setting an analysis period of $T=3600$, the hoisting gearbox lifetime dataset is divided into 2622 groups $G_{i}(i=$ $1,2, \ldots, 2622)$. The ISSE of each group is calculated separately and the threshold factor $a$ is set as two times of the standard deviation of $G_{1}$, that is $a=2 \times s$, where $s=\operatorname{std}\left(G_{1}\right), G_{1}=$ $\left[X_{1}, X_{2}, \ldots X_{T}\right]$.

The ISSE feature sequence $I_{j}$ of the hoisting gearbox during the lifetime evolution process is shown in Fig. 8a. The deeper the degradation, the larger the ISSE value with obvious phases. However, due to some strong random impact in the $R M S$ sequence, there are some obvious fluctuations in the ISSE curve which affects the accurate tracking of the performance degradation trend. The SSE curve is shown in Fig. 8b for comparison. It is evident that the curve is highly volatile and does not exhibit a trend that is consistent with the degradation process. The main reason reflects that the original symbol method is 'sensitive' to random impact, inducing the main law's annihilation in the random impact components.

The comparison of ISSE evolution curves with a different threshold factor is shown in Fig. 9. It is clear that the overall trend of the four curves does not change, while the ISSE range and phase sensitivity changes with the factor. The smaller the value of threshold factor, the smaller ISSE range, the higher the sensitivity in the initial degradation phase while the sensitivity is lower in later degradation phase. The main reason reflects that the threshold factor determines the symbol division criteria. The smaller of the value, the more sensitive it is to the symbol mode change caused by weak shock, and the lower it is to the discrimination of the mode changing caused by strong impact. The degradation process of the gearbox is accompanied by the decrease of the weak impact components and increase of strong impact components. Therefore, the value of the threshold factor will affect the sensitivity in different performance degradation phases. The threshold factor is set as 


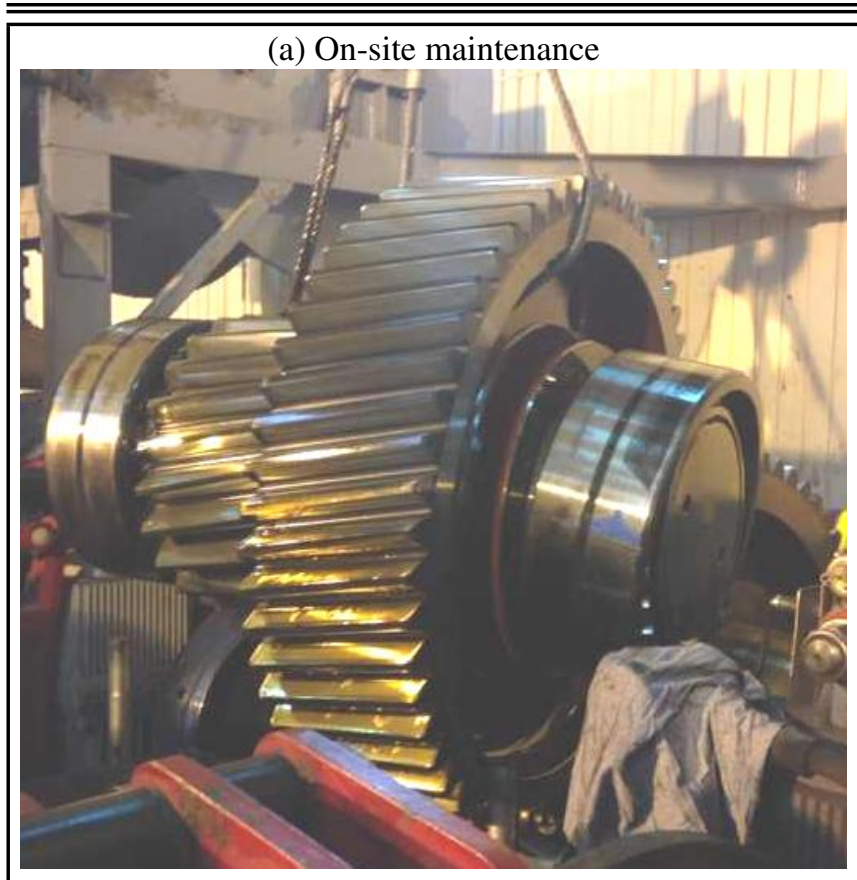

(b) Roller abrasion

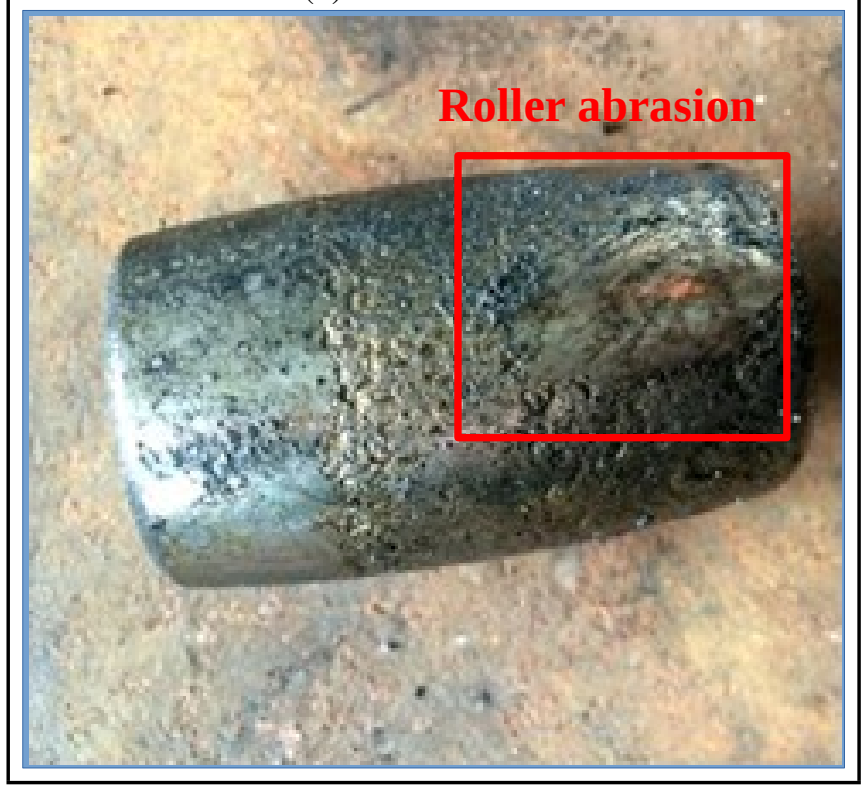

Figure 6. The maintenance of the hoisting gearbox.

$a=2 s$ in this paper for balancing the sensitivity during the whole degradation process.

The influence of parameter $m$ in ISSE analysis is shown in Fig. 10. It is evident in Fig. 10a that parameter $m$ does not affect the overall trend of ISSE curve. The symbol pattern types will increase along with the value of $m$, and then the ISSE value will increase consistently. Taking $G_{2150}$, which has the largest value in the $I S S E$ curve, as an example, the ISSE value under different values of $m$ is shown in Fig. 10b. The ISSE value is almost stable at 1 when the $m$ value is greater than 14 . Therefore, the parameter is set as $m=8$ in this paper to improve the ISSE's sensitivity to the failure mode.

\subsection{Degradation Feature Extraction Based on Online_ISSE}

Aiming at reducing the influence of random impact and improving the stability of the degradation feature, the sliding win-

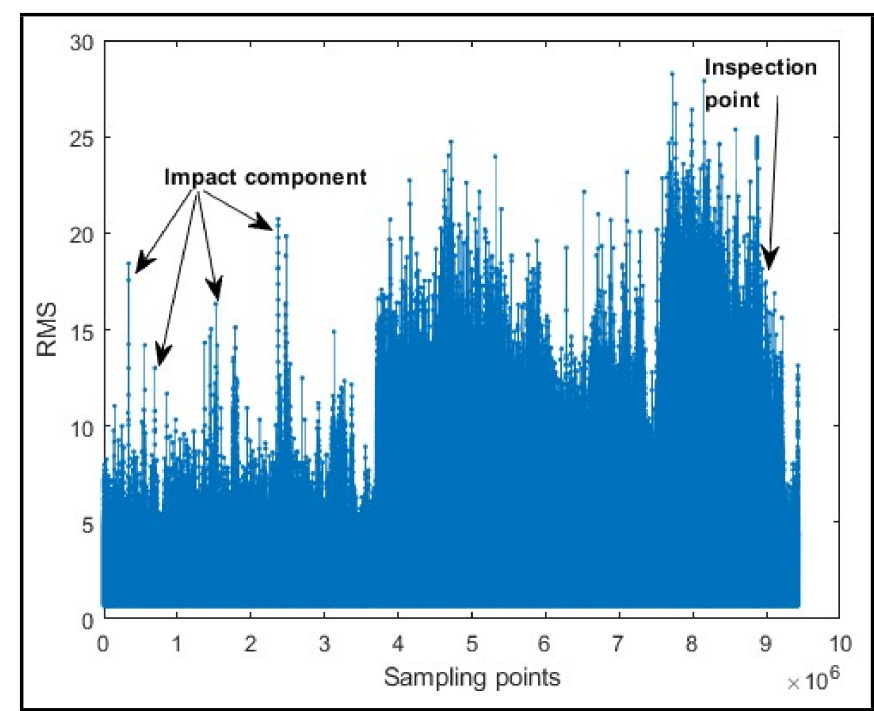

Figure 7. Lifetime vibration RMS sequence waveform.

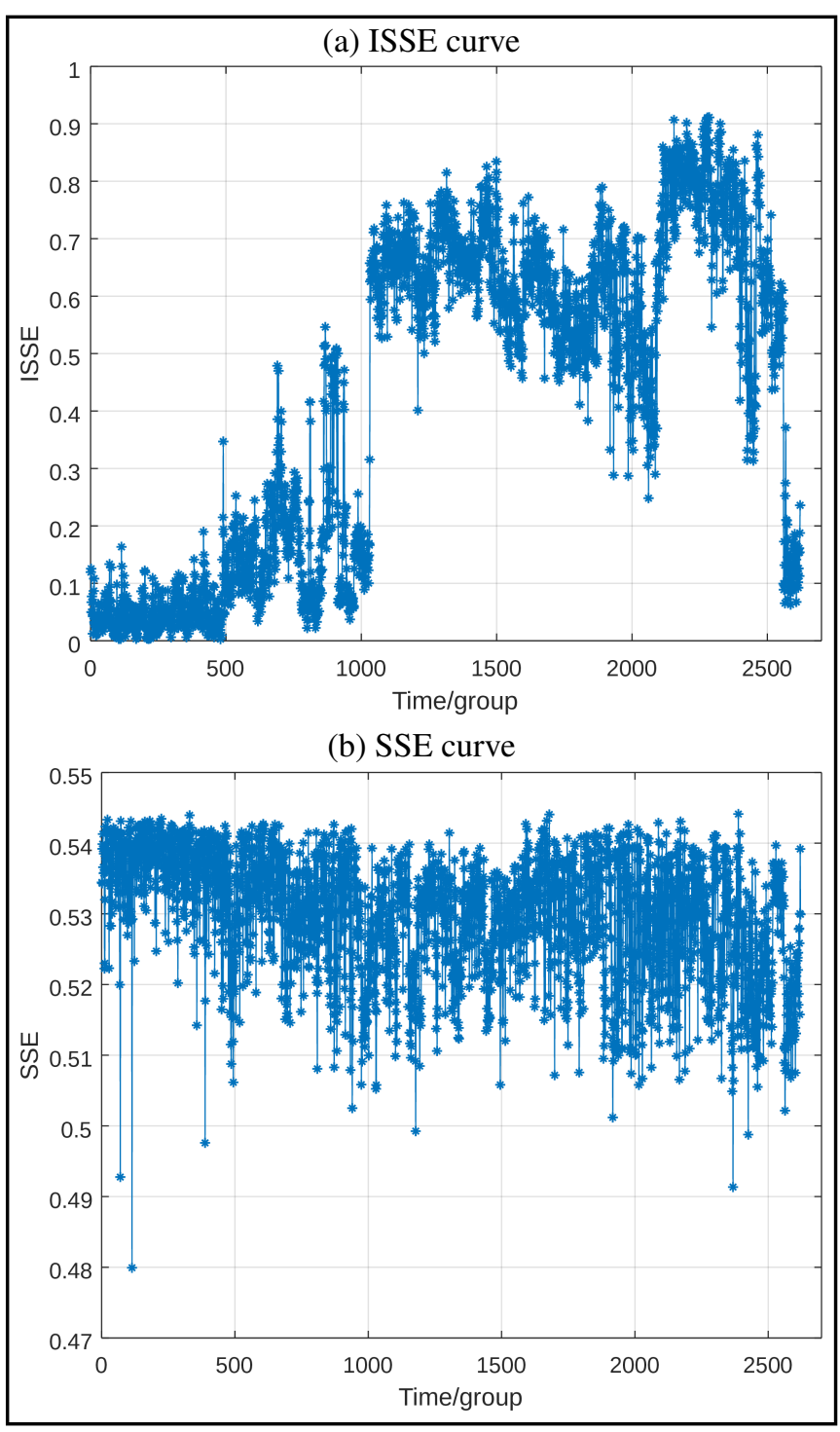

Figure 8. The contrast of different SSE curves. 


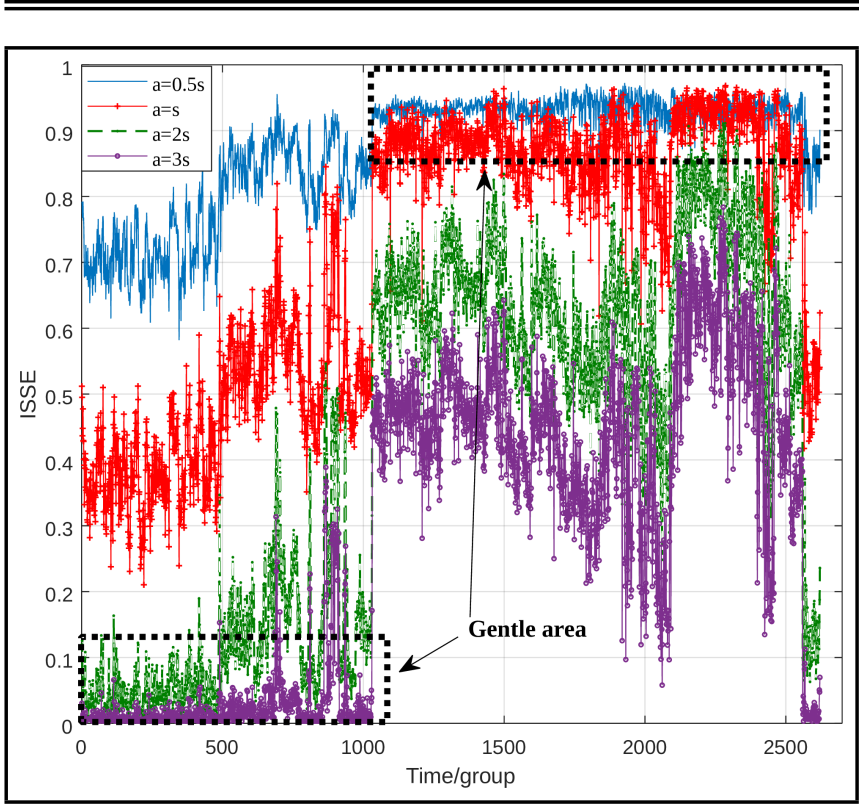

Figure 9. Influence of different threshold factor on ISSE

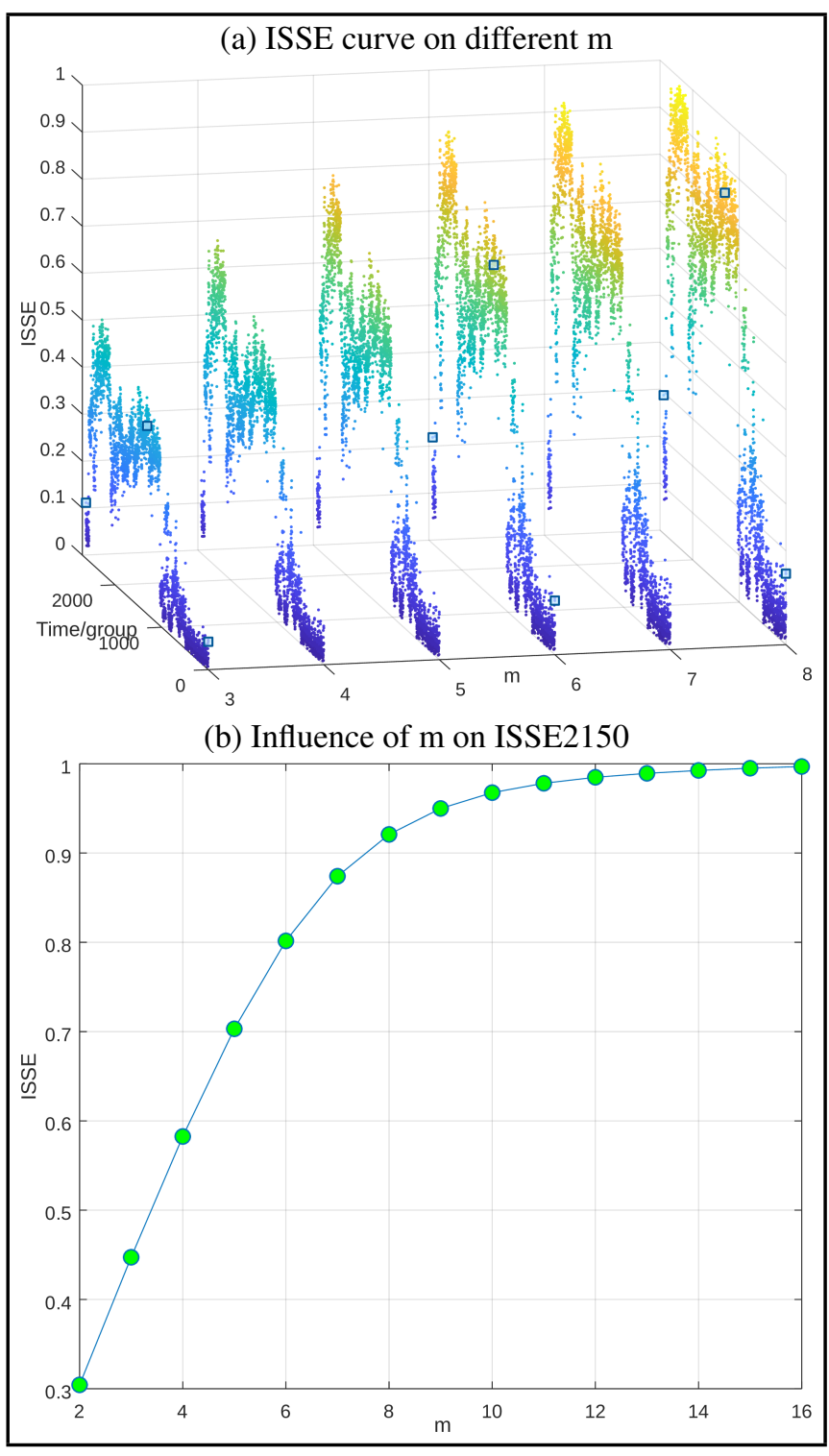

Figure 10. The influence of $m$ on ISSE value.

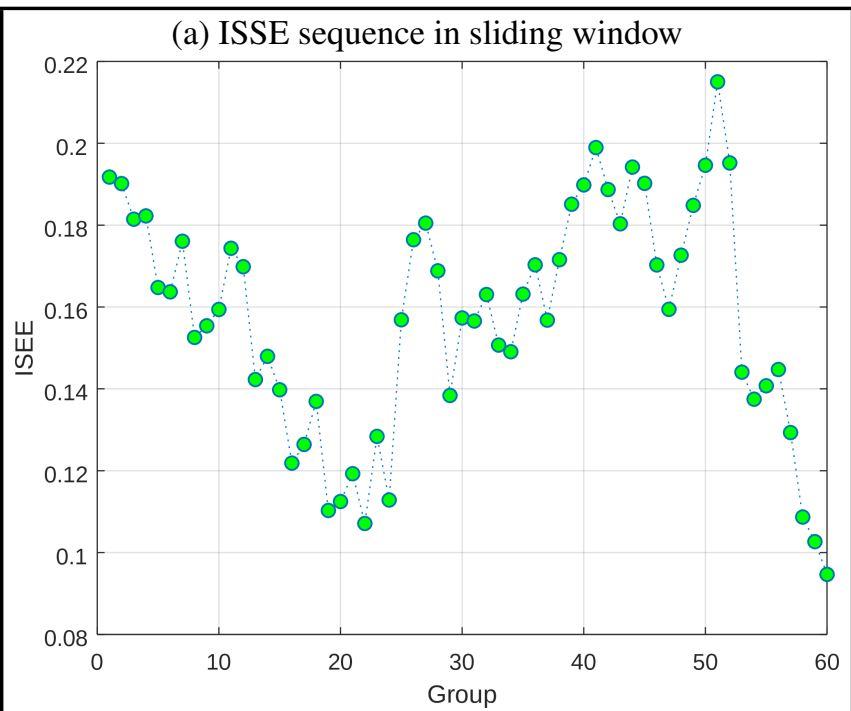

(b) Result of statistical probability

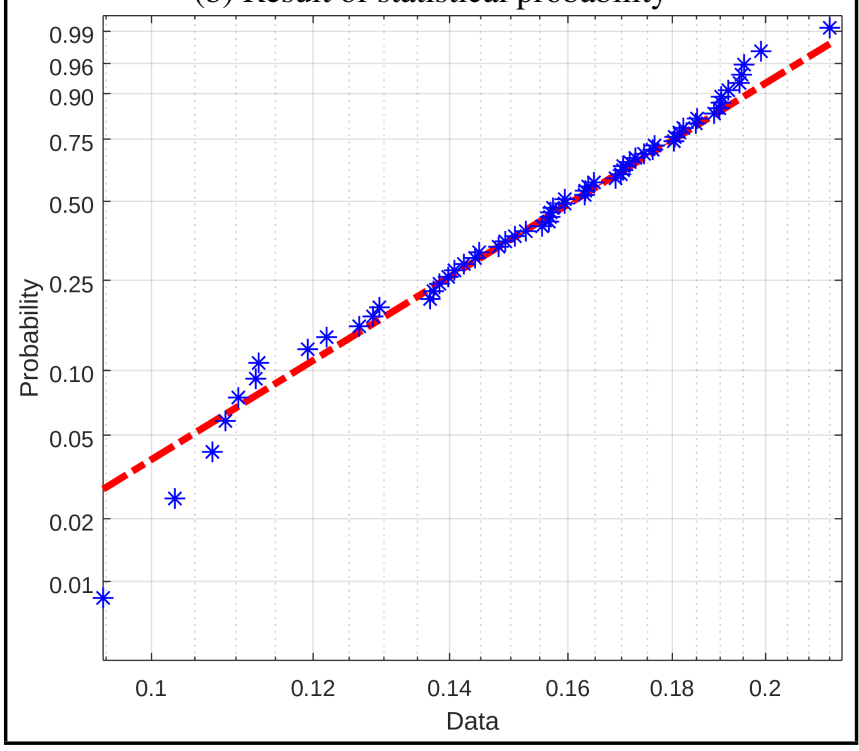

Figure 11. Weibull fitting in sliding window.

dow and the Weibull fitting are used to extract the scale factor, which is used as an online degradation feature online_ISSE. The sliding window is set as $w=60$ and $s=1$. The ISSE sequence in any sliding window is shown in Fig. 11a and the statistical rule is verified by the Weibull distribution. The result is shown in Fig. 11b. The statistical probability of this sequence is approximately linear which indicates that the ISSE sequence conforms to the Weibull distribution, and it is effective to use the Weibull fitting to analyze the statistical pattern.

The Weibull fitting is performed on each sliding window and the obtained scale parameter curve is shown in Fig. 12. It is apparent that the online ISSE curve is smoother and more stable than ISSE curve above. The influence of the impact component is filtered out. The influence of the window width parameter $w$ is shown in Fig. 13. It is evident that this parameter can affect the filtering ability on random impact components. The smaller the width, the more obvious the random fluctuation of the curve which allows more details of the curve. The larger the width, the more obvious the main curve's trend. Therefore, the appropriate sliding window width could be set according to actual needs. 


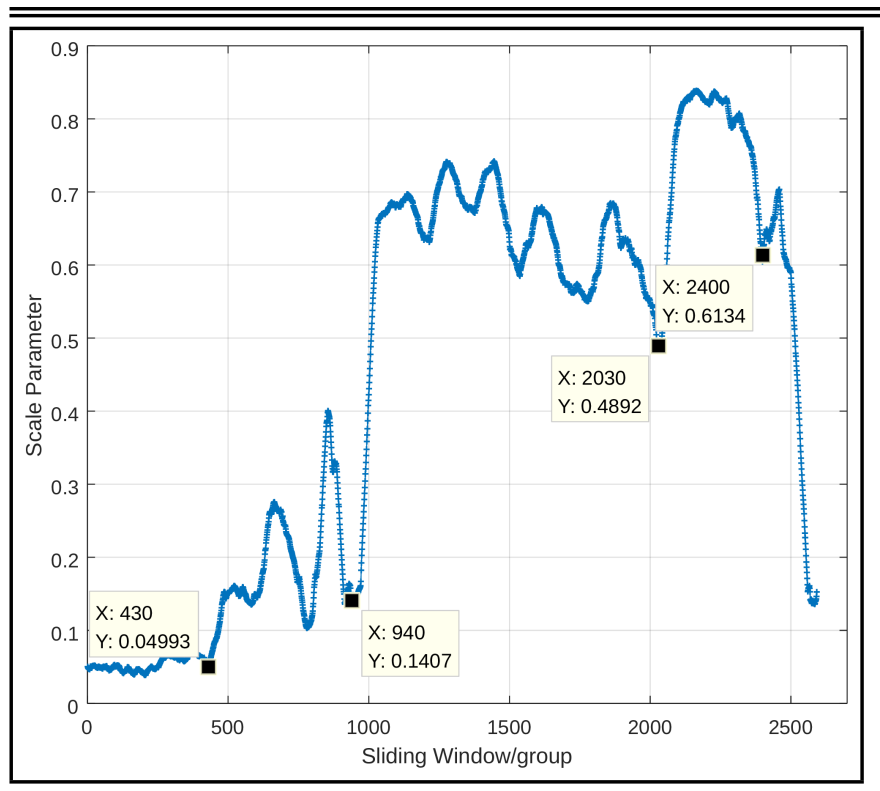

Figure 12. The degradation feature of online_ISSE.

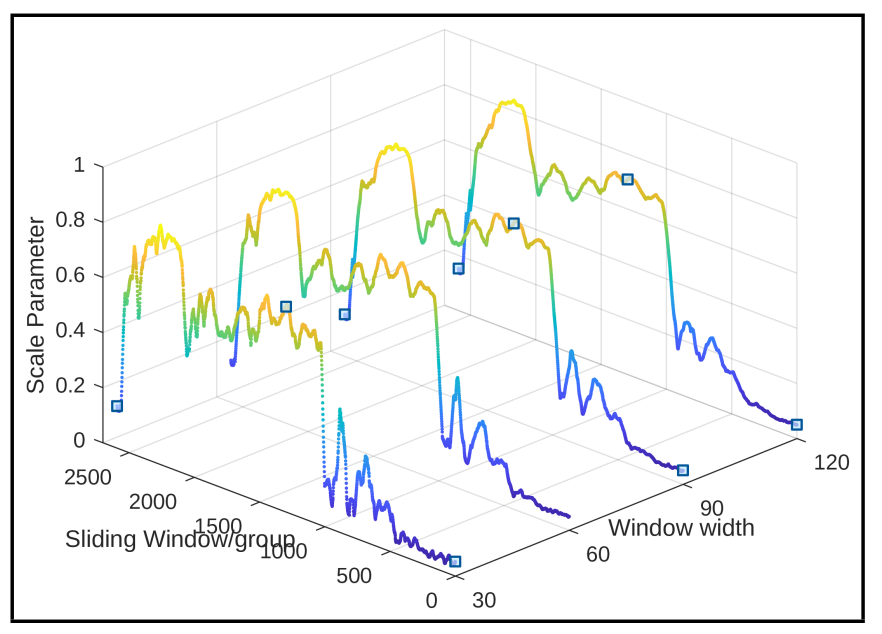

Figure 13. The influence of sliding window width on online_ISSE

\subsection{Degradation Condition Assessment}

According to the steps of the logistic regression model, the online_ISSE feature samples of health and failure conditions are selected first. We selected fifty healthy degradation features from the $1^{\text {st }}$ to the $50^{\text {th }}$ online_ISSE sequence. Pursuant to the principle above, the deeper the degradation degree, the higher the value of the online_ISSE, and when the maximum value of online ISSE is 1 , we define the degradation feature of the failure samples as online_ISSE $=1$, and then twenty groups of failure samples are selected. The health indicator $C V$ is defined as 0.95 and 0.05 respectively, some degradation features are shown in Table 2.

The selected typical seventy samples are used for the logistic regression model training and the model parameters are calculated with maximum likelihood method, $\alpha=3.2062$, $\beta=-6.1390$. The constructed logistic regression model is as follows:

$$
C V=\frac{1}{1+e^{\left(\alpha+\beta \cdot \text { online_ISSE }_{i}\right)}}, \quad(i=1,2 \ldots, 70) .
$$

Taking the online ISSE sequence as the unknown condition samples and calculating $C V$ based on the logistic regression
Table 2. Degradation feature of typical samples.

\begin{tabular}{|c|c|c|c|}
\hline Number & Condition & Online_ISSE & $\mathrm{CV}$ \\
\hline 1 & \multirow{5}{*}{ Health condition } & 0.0587 & 0.95 \\
\hline 2 & & 0.0550 & 0.95 \\
\hline 3 & & 0.0545 & 0.95 \\
\hline 4 & & 0.0549 & 0.95 \\
\hline 5 & & 0.0543 & 0.95 \\
\hline 6 & \multirow{5}{*}{ Failure condition } & 1 & 0.05 \\
\hline 7 & & 1 & 0.05 \\
\hline 8 & & 1 & 0.05 \\
\hline 9 & & 1 & 0.05 \\
\hline 10 & & 1 & 0.05 \\
\hline
\end{tabular}

model above, the obtained health indicator curve is shown in Fig. 14a. It is evident that the degradation condition of hoisting gearbox presents an overall downward trend and the degradation stages is significant. The value of $C V$ is stable around 0.94 before the $430^{t h}$ sliding window when the hoisting gearbox is considered in a healthy condition. During the $430^{t h}$ and the $940^{\text {th }}$ sliding window, the curve presents a slight deterioration with some fluctuations, and the minimum value of $C V$ is about 0.78 . This period is considered as a slight degradation condition. After about the $940^{t h}$ sliding window, the hoisting gearbox has a significant performance degradation, and the value of $C V$ drops to the interval [0.2, 0.5], and the fluctuations are also apparent at the same time. Consideration is given that the hoisting gearbox is in a severe degradation condition. After about the $2000^{\text {th }}$ sliding window, it is obvious that the curve drops and the minimum value of $C V$ reaches around 0.12 . The hoisting gearbox is defined as a failure in this period. At about the $2400^{t h}$ sliding window, the gearbox was overhauled, and the worn bearing was replaced. After a running-in period, the $C V$ value rises rapidly, and the hoisting gearbox returns to a healthy condition. A similar $C V$ curve is shown in Fig. 14b in which SSE degradation features are selected and calculated. The curve has large fluctuations due to the volatility of the ISSE feature, with which some misjudgments on degradation condition happen.

\section{CONCLUSION}

In this paper, to solve the problem of degradation assessment for a hoisting gearbox, a degradation assessment technique based on online improved symbol sequence entropy (online ISSE) and a logistic regression model was proposed. The lifetime vibration signal of a hoisting gearbox was introduced for instance analysis. The following conclusions were obtained.

(1) The improved symbol sequence entropy algorithm introduces a threshold factor, which not only retains the direction changing information, but also depicts "coarse graining" amplitude changing information. Compared to the symbol sequence entropy, the $I S S E$ algorithm was able to exploit the complexity evolution law in the performance degradation process of the hoisting gearbox.

(2) The influence of fluctuations in the ISSE sequence was able to be filtered combing the sliding window with the Weibull fitting method, thus improving the stability and forming the degradation feature named online ISSE, which can track the performance degradation condition more accurately.

(3) Combining online_ISSE with the logistic regression model, the health factor of the unknown signal can be calculated online to assess its performance degradation condition. 


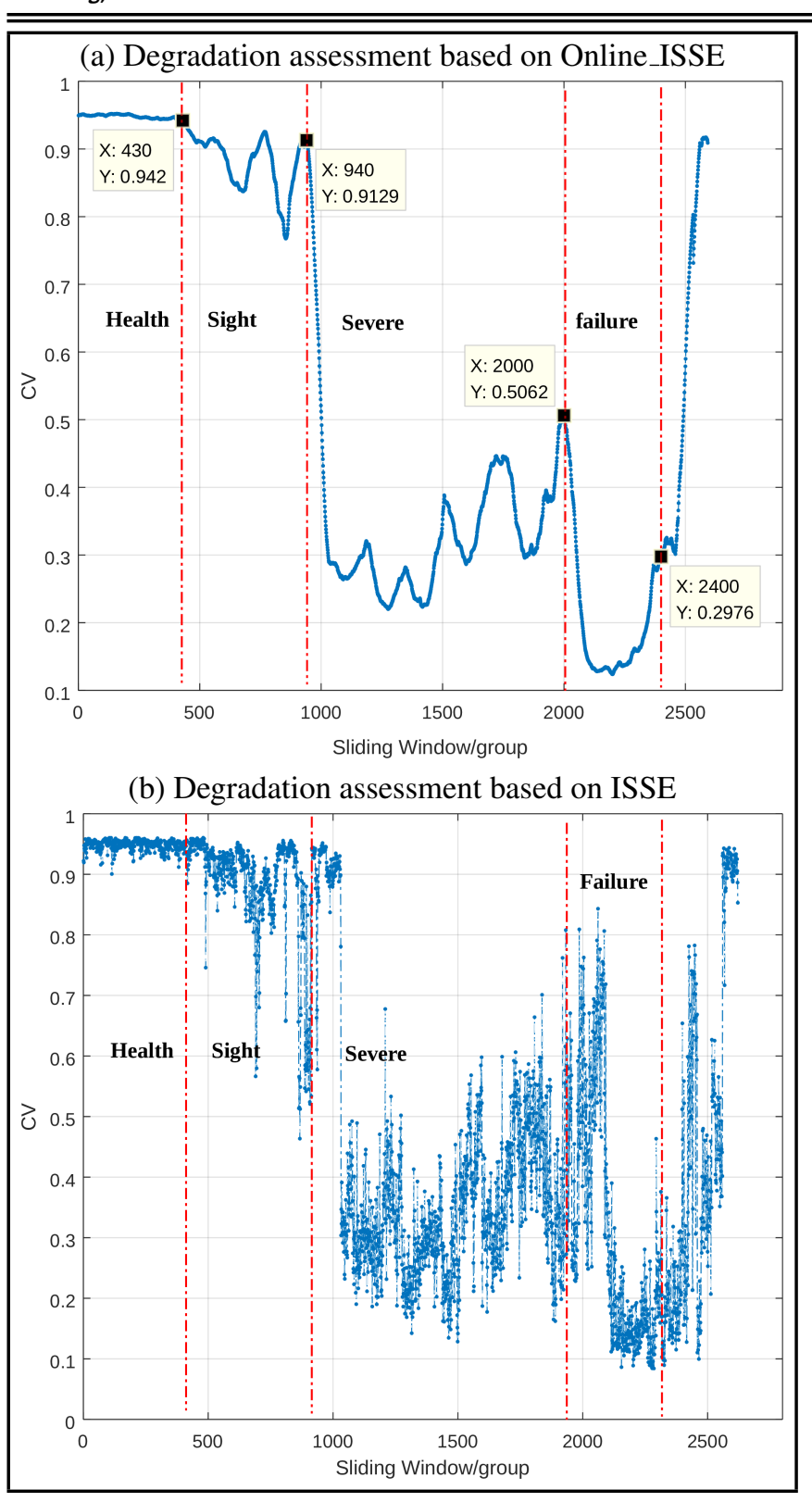

Figure 14. Degradation assessment of hoisting gearbox.

(4) Compared to some proposed vibration analysis methods as preferences, ${ }^{31-33}$ the technique proposed in this paper was featuring a hoisting gearbox in the quay crane which had a typical nonlinear nonstationary and aperiodic character. Some proposed vibration analysis methods will not be suitable for this type of signal in the quay crane.

(5) How to decide the number and boundaries of the degradation conditions will be the focus in a future analysis.

\section{ACKNOWLEDGMENT}

The authors declare no conflict of interest in preparing this article

\section{REFERENCES}

1 Tan, C., Yan W., Yue, J., Quay crane scheduling in automated container terminal for the trade-off between operation efficiency and energy consumption, Advanced Engineering Informatics., 48(1), 101285-101294. (2021). https://dx.doi.org/10.1016/j.aei.2021.101285
2 Dimitris M., Ekaterini V., A cloud-based cyberphysical system for adaptive shop-floor scheduling and condition-based maintenance, Journal of Manufacturing Systems., 47(2), 179-198, (2019). https://dx.doi.org/10.1016/j.jmsy.2018.05.008

3 Dhamande, L. S., Chaudhari M. B., Compound Gear-Bearing Fault Feature Extraction Using Statistical Features Based On Time-Frequency Method, Measurement., 125, 63-77, (2018). https://dx.doi.org/10.1016/j.measurement.2018.04.059

4 Wang, L. H., Zhao, X. P., Wu, J. X., et al., Motor Fault Diagnosis Based on Short-time Fourier Transform and Convolutional Neural Network, Chinese Journal of Mechanical Engineering., 30(6), 1357-1368, (2017). https://dx.doi.org/10.1007/s10033-017-0190-5

5 Glowacz, A., Glowacz, W., Glowacz, Z., et al., Early fault diagnosis of bearing and stator faults of the single-phase induction motor using acoustic signals, Measurement., 113, 1-9, (2018). https://dx.doi.org/10.1016/j.measurement.2017.08.036

6 Antoni, J., Randall, R. B., The spectral kurtosis: application to the vibratory surveillance and diagnostics of rotating machines, Mechanical Systems \& Signal Processing., 20(2), 308-331, (2006). https://dx.doi.org/10.1016/j.ymssp.2004.09.002

7 Randall, R. B., Antoni, J. , Rolling element bearing diagnostics - A tutorial, Mechanical Systems \& Signal Processing., 25(2), 485-520, (2011). https://dx.doi.org/10.1016/j.ymssp.2010.07.017

8 Wang, Dong. Spectral, L2 / L1, norm: A new perspective for spectral kurtosis for characterizing non-stationary signals, Mechanical Systems and Signal Processing., 104, 290-293, (2018). https://dx.doi.org/10.1016/j.ymssp.2017.11.013

9 Araneo, R., Attolini, G., Celozzi, S., et al., TimeDomain Shielding Performance of Enclosures: A Comparison of Different Global Approaches, IEEE Transactions on Electromagnetic Compatibility., 58(2), 434-441, (2016). https://dx.doi.org/10.1109/TEMC.2015.2497742

${ }^{10}$ Li, Y., Yang, Y., Li, G., et al., A fault diagnosis scheme for planetary gearboxes using modified multi-scale symbolic dynamic entropy and mRMR feature selection, Mechanical Systems and Signal Processing., 91, 295-312, (2017). https://dx.doi.org/10.1016/j.ymssp.2016.12.040

11 Han, M., Pan, J., A fault diagnosis method combined with LMD, sample entropy and energy ratio for roller bearings, Measurement., 76, 7-19, (2015). https://dx.doi.org/10.1016/j.measurement.2015.08.019

12 Jomaa, M., Bogaert, P. V., Jrad, N., et al., Multivariate improved weighted multiscale permutation entropy and its application on EEG data, Biomedical 
signal processing and control., 52(7), 420-428, (2019). https://dx.doi.org/10.1016/j.bspc.2018.08.004

${ }^{13} \mathrm{Li}$, Y., Gao, Q., Miao, B., et al., Application of the refined multiscale permutation entropy method to fault detection of rolling bearing, Journal of the Brazilian Society of Mechanical Sciences and Engineering.,43(5), 1-14, (2021). https://dx.doi.org/10.1007/s40430-021-02986-7

${ }^{14}$ Sun, D. J., Wang, B., Hu, X., et al. , An Online Degradation Feature Extraction Technique for Shore Bridge Gearbox Based on Morphological Fractal Dimension and Sliding Window Weibull Fitting, Shock and Vibration., 2019, 1-9, (2019). https://dx.doi.org/10.1155/2019/9216809

15 Li, Y., Yang, Y., Wang, X., et al. Early fault diagnosis of rolling bearings based on hierarchical symbol dynamic entropy and binary tree support vector machine, Journal of Sound and Vibration., 428, 72-86, (2018). https://dx.doi.org/10.1016/j.jsv.2018.04.036

16 Li, H. L., Wang, J., Deng, B., et al. Analysis of mutual information and synchronism for epileptic EEG signals., Computer Engineering and Applications., 49(6), 1922, (2013).

17 Du, F., Wang, S. T., Dai, J. F., et al., Multiscale sign series entropy analysis based on the young and middle-aged electroencephalogram, Beijing Biomedical Engineering., 35(6), 599-603, (2016).

18 Shen, F., Dai, G., Lin, G., et al., EEG-based emotion recognition using 4D convolutional recurrent neural network. Cognitive Neurodynamics., 14(6), 1-14, (2020). https://dx.doi.org/10.1007/s11571-020-09634-1

19 Ban, H., Wang, D., Wang, S., et al., Multilocation and Multiscale Learning Framework with Skip Connection for Fault Diagnosis of Bearing under Complex Working Conditions, Sensors., 21(9), 3226-3240, (2021). https://dx.doi.org/10.3390/s21093226

20 Bharathi, R., Selvarani, R., Hidden Markov Model Approach for Software Reliability Estimation with Logic Error. International Journal of Automation and Computing., 17(2), 305-320, (2020). https://dx.doi.org/10.1007/s11633019-1214-7

${ }^{21}$ Caesarendra, W., Widodo, A., Yang, B. S., Application of relevance vector machine and logistic regression for machine degradation assessment, Mechanical Systems \& Signal Processing., 24(4), 1161-1171, (2010). https://dx.doi.org/10.1016/j.ymssp.2009.10.011

${ }^{22}$ Choi, M. C. H., On resistance distance of Markov chain and its sum rules. Linear Algebra \& Its Applications., 571, 14 25, (2019). https://dx.doi.org/10.1016/j.laa.2019.02.014

${ }^{23}$ Fantoukh, N. I., Ismail, M., Bchir, O., Automatic Determination of the Number of Clusters for Semi-Supervised Relational Fuzzy Clustering, International Journal of Fuzzy
Logic and Intelligent Systems., 20(2), 156-167, (2020). https://dx.doi.org/10.5391/IJFIS.2020.20.2.156

${ }^{24}$ Mc, A., Ar, B., Rm, C., Symbolic transfer entropy test for causality in longitudinal data, Economic Modelling., 94, 649-661, (2021). https://dx.doi.org/10.1016/j.econmod.2020.02.007

25 Aririguzo, J. C., Ekwe, E. B., Weibull distribution analysis of wind energy prospect for Umudike, Nigeria for power generation, Robotics and ComputerIntegrated Manufacturing., 55(B), 160-163, (2019). https://dx.doi.org/10.1016/j.rcim.2018.01.001

${ }^{26}$ Zhang, Y. G., Wang, P. H., Zhang, X., et al. Synthetic fault factor features under Weibull stochastic interference, International Journal of Electrical Power \& Energy Systems., 77, 19-24, (2016). https://dx.doi.org/10.1016/j.ijepes.2015.11.033

${ }^{27} \mathrm{Li}$, Zhang, Jingyuan, et al. Performance analysis of circle polarization shift keying modulation over the exponentiated Weibull distribution, Optical Review., 27(1), 39-44, (2020). https://dx.doi.org/10.1007/s10043-019-00562-y

28 Faisal, M., Scally, A., Howes, R., et al. A comparison of logistic regression models with alternative machine learning methods to predict the risk of in-hospital mortality in emergency medical admissions via external validation, Health informatics journal., 26(1), 34-44, (2020). https://dx.doi.org/10.1177/1460458218813600

${ }^{29}$ Hajipour, F., Jozani, M. J., Moussavi, Z., A comparison of regularized logistic regression and random forest machine learning models for daytime diagnosis of obstructive sleep apnea, Medical \& Biological Engineering \& Computing., 58(10), 1-13, (2020). https://dx.doi.org/10.1007/s11517020-02206-9

30 Tang, G., Li, J. X., Hu, X., Accurate Approximate Clustering Algorithm and Its Application in the State Monitoring of Crane, Journal of Donghua University, Natural Sciences., 44(4), 590-594, (2018).

31 Caesarendra, W., Pratama, M., Kosasih, B., et al. Parsimonious Network based on Fuzzy Inference System (PANFIS) for Time Series Feature Prediction of Low Speed Slew Bearing Prognosis, Applied Sciences., 8(12), 2656-2665, (2018). https://dx.doi.org/10.3390/app8122656

32 Glowacz, A., Glowacz, W., Kozik, J., et al. Detection of Deterioration of Three-phase Induction Motor using Vibration Signals, Measurement Science Review.,19(6), 241249, (2019). https://dx.doi.org/10.2478/msr-2019-0031

33 Glowacz, A., Acoustic fault analysis of three commutator motors, Mechanical Systems and Signal Processing., 133(1), 106226-106230. (2019). https://dx.doi.org/10.1016/j.ymssp.2019.07.007 